\title{
Evidence-based treatment of patients with rectal cancer (Review)
}

\author{
QIANG ZHANG, JIE YANG and QUN QIAN
}

\author{
Department of General Surgery, Xiangyang Hospital Affiliated to Hubei University of Medicine, \\ Xiangyang, Hubei 441000, P.R. China
}

Received September 10, 2015; Accepted January 11, 2016

DOI: $10.3892 / 01.2016 .4100$

\begin{abstract}
Rectal cancer is a worldwide disease whose incidence has increased significantly. Evidence-based medicine is a category of medicine that optimizes decision making by using evidence from well-designed and conducted research. Evidence-based medicine can be used to formulate a reasonable treatment plan for newly diagnosed rectal cancer patients. The current review focuses on the application of evidence-based treatment on patients with rectal cancer. The relationship between perioperative blood transfusion and recurrence of rectal cancer after surgery, the selection between minimally invasive laparoscopic surgery and traditional laparotomy, choice of chemotherapy for patients with rectal cancer prior to surgery, selection between stapled and hand-sewn methods for colorectal anastomosis during rectal cancer resection, and selection between temporary ileostomy and colostomy during the surgery were addressed. Laparoscopy is considered to have more advantages but is time-consuming and has high medical costs. In addition, laparoscopic rectal cancer radical resection is preferred to open surgery. In radical resection surgery, use of a stapling device for anastomosis can reduce postoperative anastomotic fistula, although patients should be informed of possible anastomotic stenosis.
\end{abstract}

\section{Contents}

1. Introduction

2. Relationship between perioperative blood transfusion and recurrence of rectal cancer following surgery

3. Selection between minimally invasive laparoscopic surgery and traditional laparotomy

4. Choice of chemotherapy for patients with colorectal cancer prior to surgery

5. Selection between stapled and hand sewn methods for colorectal anastomosis during rectal cancer resection

Correspondence to: Dr Jie Yang, Department of General Surgery, Xiangyang Hospital Affiliated to Hubei University of Medicine, 15 Jiefang Road, Xiangyang, Hubei 441000, P.R. China

E-mail: 1692710644@qq.com

Key words: rectal cancer, evidence-based treatment, review literature
6. Selection between temporary ileostomy and colostomy during surgery

7. Application of evidence-based medical evidence for treatment plan

\section{Introduction}

The application of evidence-based medicince has reached a stage where theory is used to guide clinical practice. Rectal cancer is a worldwide disease whose incidence has increased significantly in recent years (1-3). Clinical randomized controlled trials (RCTs) associated with the comprehensive treatment of rectal cancer have been conducted by various international research groups, thereby increasing the treatment level of rectal cancer (1-3). The current review summarizes the progress of rectal cancer treatment.

A search of the Cochrane Library (2008 phase), EMBASE (1998-2011), Blackwell electronic journals (1998-2011) and Elsevier electronic journals (1998-2011) identified 5 articles regarding system evaluation, 34 articles concerning RCTs, 5 articles regarding meta-analysis, 10 articles concerning cohort studies, and 7 articles regarding clinical observations and experiments $(4,5)$. Quality assessment was conducted on the obtained evidence. A systematic evaluation of those articles was obtained from the Cochrane Library and the included RCTs yielded randomized study results, using a blind analysis. The follow up was relatively complete, the results demonstrated the treatment analysis employed, and due to the control of confounding factors of the results, the conclusions obtained had a certain authenticity. Articles in the RCTs cited in the current review were derived from a multicenter study (6). The sample size was relatively large, intensity of argumentation was relatively strong although some RCTs contained a smaller sample size, or included a limited number of cases. Although the specific random method was mentioned, it was not sufficiently elucidated, and whether allocation concealment was carried out was not clear. In addition, whether the baseline was balanced, or whether the blind method was utilized was also unclear.

\section{Relationship between perioperative blood transfusion and recurrence of rectal cancer after surgery}

Of the 36 RCT studies collated and analyzed by Amato and Pescatori, including 12,127 patients (7), the findings of 23 studies showed that perioperative blood transfusion had 
a decisive impact on recurrence of rectal cancer following surgery. Twenty-two studies employed multivariate analysis, and the findings of 14 of the 22 studies indicated that perioperative transfusion served as an independent prognostic factor in the recurrence of colorectal cancer after surgery. The value of the comprehensive evaluation of perioperative blood transfusion causing recurrence of colorectal cancer was 1.42 [95\% confidence interval (CI): 1.20-1.67], as confirmed by a stratified meta-analysis (8). The analysis results supported that the perioperative transfusion caused a harmful acceleration effect on the recurrence of rectal cancer. However, due to error in the observed cases and the effect of surgical operations, no causal link between perioperative blood transfusion and the recurrence of colorectal cancer has been established. Thus, indications of a blood transfusion therapy for a patient with rectal cancer should be stringently limited. McAlister et al (9) conducted a meta-analysis on six RCT studies and two prospective studies with good control during the same period, and concluded that the odds ratio (OR) value of tumor recurrence following transfusion was 1.06 (95\% CI: $0.88-1.28$ ). However, there was not sufficient evidence to prove that perioperative allogeneic blood transfusion was capable of increasing the occurrence of severe surgical complications such as tumor metastasis. The prospective randomized study covering 140 cases carried out by Ray-Coquard et al (10) identified that tumor metastasis and the recurrence rate following surgery between the group receiving perioperative transfusion of red blood cell suspension and the group using blood substitutes and erythropoietin group were significantly different. The authors of that study determined that perioperative blood transfusion was highly correlated with the recurrence of rectal cancer after surgery.

\section{Selection between minimally invasive laparoscopic surgery and traditional laparotomy}

Of the 48 studies covering 4,224 patients collated and analyzed by Rosenberg et al (11), 3 studies conformed to single-center RCT of $1 b$ level of evidence, 12 studies conformed to single-cohort study of $2 \mathrm{~b}$ level of evidence, 5 studies complied with single-center case-control studies of $3 \mathrm{~b}$ level of evidence and 28 case reports complied with a grade 4 level of evidence. Only one RCT identified 3- and 5-year disease-free survival, and no meta-analysis was performed. Authors of that study indicated that with regard to minimally invasive laparoscopic surgery and traditional laparotomy for colorectal cancer, the differences in postoperative survival, local recurrence, mortality, and the occurrence of anastomotic fistula, the length of the lesion margins, detection and prevention of lymph node metastases or lack thereof were not significant. Evidence showed that laparoscopic surgery is beneficial owing to less bleeding, faster diet recovery after surgery, use of fewer anesthetics for postoperative analgesia and less postoperative immune response. Time-consuming surgery and high medical costs constituted its drawbacks. No studies on the quality of life after laparoscopic surgery have been previously conducted. This comprehensive analysis of the results was primarily derived from a non-randomized study (12), thus, the short-term advantages of laparoscopic surgery were evident. However, no conclusion was drawn on the endpoint criteria of oncology mortality. Thus, a large randomized controlled multicenter study is needed to confirm the results.

In a systematic review Reza et al identified that patients recovered more rapidly with reduced suffering following minimally invasive laparoscopic surgery (13). However, there was no difference in terms of overall mortality and postoperative complications compared with laparotomy. RCT on cases of laparoscopic-assisted radical resection of rectal carcinoma and laparotomy conducted by Lacy et al (14) showed that minimally invasive laparoscopic surgery significantly reduced postoperative pain index of patient and significantly improve quality of life scores. RCT carried out by Cheung et al (15) and Kuhry et al (16), respectively, suggested that laparoscopic surgery had the advantage of less bleeding, faster diet recovery following surgery, and fewer anesthetics for postoperative analgesia. Through cohort studies, Larson et al (17) suggested that the safety of colon-anal anastomosis in assisted laparoscopic radical resection of rectal carcinoma overmatched that of open surgery, and the incidence of anastomotic fistula was reduced significantly. RCT conducted by Buunen et al (18) found that the difference of the 5-year survival between the laparoscopic-assisted resection of rectal cancer group and the laparotomy group was significantly different. Thus, they concluded that laparoscopic-assisted radical resection of rectal cancer is the preferred radical treatment for rectal cancer.

\section{Choice of chemotherapy for patients with colorectal cancer prior to surgery}

Cammà et al (19) performed a meta-analysis on 14 RCT studies, and the results showed that compared with single radical treatment, preoperative radiotherapy for rectal cancer in combination with radical surgical resection significantly reduced the 5-year local recurrence rate and overall mortality. The OR identified was 0.71 (95\% CI: $0.61-0.82 ; \mathrm{p}<0.001)$ and the OR of the local recurrence rate was 0.49 (95\% CI: 0.38-0.62; p<0.001). The incidence of distant metastasis between the two groups was not statistically significant. Thus, it was concluded that for patients with rectal cancer suitable for surgical resection, radiation therapy prior to surgery significantly improved the survival rate. In a meta-analysis conducted by Kosmider et al (20), it was concluded that preoperative radiotherapy reduced the mortality of tumor recurrence. The systematic review carried out by Prosnitz et al (21) did not provide a final conclusion on preoperative radiotherapy quality control. A randomized controlled double-blind study conducted by Machiels et al (22) and Kapiteijn et al (23) suggested that preoperative radiotherapy of rectal cancer reduced the tumor stage and local recurrence rate, and improved the ratio of rectal preservation surgery.

The FFCD 9203 study by Gérard et al (24), which identified 733 cases of patients with digital rectal examination palpable T3-4 stage rectal cancer were randomly and double-blindly divided into th epreoperative radiotherapy and postoperative radiotherapy groups. The results of that study showed that compared with the postoperative radiotherapy group, in the preoperative radiotherapy group, the pathological complete response rate increased $(11.4$ vs. $3.6 \%, \mathrm{p}<0.05)$ and the rate of lower stage of tumor (58.6 vs. $30.0 \%$, p<0.05). 


\section{Selection between stapled and hand sewn methods for colorectal anastomosis during rectal cancer resection}

Choy et al (25) analyzed six clinical studies including 955 patients with rectal cancer resection anastomosis. The meta-analysis results of 3 RCT with the largest sample size showed that compared with the manual anastomosis, stapling anastomosis significantly reduced the incidence of anastomotic fistula, OR: $0.34(0.14,0.82 ; \mathrm{p}=0.02)$. In a subgroup analysis of 825 rectal cancer patients, the results showed that compared with manual suture, stapled suturing significantly decreased the incidence of anastomotic fistula, OR: $0.28(0.10,0.75 ; \mathrm{p}=0.01)$. Following a systematic review of RCT covering 1,233 patients, Gianotti et al (26) and Lustosa et al (27) concluded that the likelihood of anastomotic stricture increased after stapling and that the operation time of manual anastomosis was relatively increased.

\section{Selection between temporary ileostomy and colostomy during surgery}

In a RCT covering 150 elderly patients, Moran et al (28) identified thatfollowing rectal cancerradical resection, the incidence of anastomotic fistula caused by temporary colostomy was significantly lower than that of ileostomy $(\mathrm{p}<0.01)$. Findings by Walker et al (29) and Arguedas et al (30) were identical to those of Moran et al (28), albeit the aforementioned three sets of data came from a single center. The level of evidence was relatively low; thus, well-designed multicenter RCTs with a large sample size are needed to confirm this conclusion. In a meta-analysis of 5 RCT studies, Lertsithichai and Rattanapichart (31) concluded that for patients who were able to undergo rectal cancer radical resection, a temporary colostomy would cause more infections and traumatic complications of the anastomotic stoma. If ileostomy is carried out, then the secondary fistula-closing surgery would cause discomfort to patients as well as an increase in treatment costs and extended treatment cycle. Through early postoperative testing and re-evaluation studies, Juneja et al (32) suggested that the incidence of anastomotic fistula caused by temporary colostomy and ileostomy following rectal cancer radical resection was not statistically different. The present review is limited by complications caused during the two surgeries, in terms of fistula. Tsikitis et al (33) suggested that for low anastomotic stoma that are $6 \mathrm{~cm}$ below the anal brink, prophylactic stoma should be conducted. For elderly patients of advanced age and poor nutrition, especially patients receiving preoperative radiotherapy, preventive purposes stoma is recommended.

\section{Application of evidence-based medical evidence for treatment plan}

Current evidence for patients with rectal cancer suggests that blood substitutes such as plasma substitute or dextran are considered ideal to minimize the quantity of surgical bleeding and avoid perioperative blood transfusion (34). Transfusion indications where required should be controlled stringently. Laparoscopic surgery has the advantage of less bleeding, faster diet recovery after surgery, fewer anesthetics for postoperative analgesia and less postoperative immune response. However, long operation time and high medical costs are its drawbacks. Taking all the above into consideration, and provided that the economic condition of the patient permits, laparoscopic rectal cancer radical resection should be employed, while open surgery is not recommended. Given that preoperative radiotherapy can significantly reduce overall mortality 5 years after the surgery, the Department of Radiotherapy should be required to make group consultations a month prior to the surgery and to formulate a preoperative pelvic radiotherapy plan to conduct preoperative radiotherapy. In radical resection surgery, adopting the stapling device for anastomosis can reduce postoperative anastomotic fistula, although patients should be informed of possible anastomotic stenosis. In the process of radical resection surgery, some patients with special conditions require temporary fecal diversion, in which case either colostomy or ileostomy may be used with equal efficiency.

\section{References}

1. Vannelli A, Basilico V, Zanardo M, Caizzone A, Rossi F, Battaglia L and Scaramuzza D: Pelvic lymphedema in rectal cancer: a magnetic resonance feasibility study: a preliminary report. Eur Rev Med Pharmacol Sci 17: 929-935, 2013.

2. Yimei J, Ren Z, Lu X and Huan Z: A comparison between the reference values of MRI and EUS and their usefulness to surgeons in rectal cancer. Eur Rev Med Pharmacol Sci 16: 2069-2077, 2012

3. Kuran S, Ozin Y, Nessar G, Turhan N and Sasmaz N: Is endorectal ultrasound still useful for staging rectal cancer? Eur Rev Med Pharmacol Sci 18: 2857-2862, 2014.

4. Liang YC, Li GX, Chen PY, et al: Laparoscopic versus conventional open resection for colorectal cancer: a meta-analysis on recurrence. Zhonghua Wei Chang Wai Ke Za Zhi 11: 414-420, 2008.

5. Tan E, Gouvas N, Nicholls RJ, et al: Diagnostic precision of carcinoembryonic antigen in the detection of recurrence of colorectal cancer. Surg Oncol 9: 115-118, 2008.

6. Purkayastha S, Tekkis PP, Athanasiou T, et al: Diagnostic precision of magnetic resonance imaging for preoperative prediction of the circumferential margin involvement in patients with rectal cancer. Colorectal Dis 9: 402-411, 2007.

7. Amato A and Pescatori M: Perioperative blood transfusions for the recurrence of colorectal cancer. Cochrane Database Syst Rev 25: CD005033, 2006.

8. Vamvakas EC and Blajchman MA: Transfusion-related mortality: the ongoing risks of allogeneic blood transfusion and the available strategies for their prevention. Blood 113: 3406-3417, 2009.

9. McAlister FA, Clark HD, Wells PS and Laupacis A: Perioperative allogeneic blood transfusion does not cause adverse sequelae in patients with cancer: A meta-analysis of unconfounded studies. Br J Surg 85: 171-178, 1998.

10. Ray-Coquard I, Dussart S, Goillot C, Mayeur D, Debourdeau P, Ghesquieres H, Bachelot $\mathrm{T}$, Le Cesne A, Anglaret B, Agostini C, et al: A risk model for severe anemia to select cancer patients for primary prophylaxis with epoetin alpha: a prospective randomized controlled trial of the ELYPSE study group. Ann Oncol 20: 1105-1112, 2009.

11. Rosenberg J, Harvald T and Haglind E: Laparoscopic versus open total mesorectal excision for rectal cancer. Ugeskr Laeger 169: 3652-3655, 2007 (In Danish).

12. Anderson C, Uman G and Pigazzi A: Oncologic outcomes of laparoscopic surgery for rectal cancer: a systematic review and meta-analysis of the literature. Eur J Surg Oncol 34: 1135-1142, 2008.

13. Reza MM, Blasco JA, Andradas E, Cantero R and Mayol J: Systematic review of laparoscopic versus open surgery for colorectal cancer. Br J Surg 93: 921-928, 2006.

14. Lacy AM, Delgado S, Castells A, Prins HA, Arroyo V, Ibarzabal A and Pique JM: The long-term results of a randomized clinical trial of laparoscopy-assisted versus open surgery for colon cancer. Ann Surg 248: 1-7, 2008. 
15. Cheung HY, Chung CC, Tsang WW, Wong JC, Yau KK and Li MK: Endolaparoscopic approach vs conventional open surgery in the treatment of obstructing left-sided colon cancer: a randomized controlled trial. Arch Surg 144: 1127-1132, 2009.

16. Kuhry E, Schwenk W, Gaupset R, Romild U and Bonjer J: Long-term outcome of laparoscopic surgery for colorectal cancer: a cochrane systematic review of randomised controlled trials. Cancer Treat Rev 34: 498-504, 2008.

17. Larson DW, Cima RR, Dozois EJ, Davies M, Piotrowicz K Barnes SA, Wolff B and Pemberton J: Safety, feasibility, and short-term outcomes of laparoscopic ileal-pouch-anal anastomosis: a single institutional case-matched experience. Ann Surg 243: 667-672, 2006.

18. Colon Cancer Laparoscopic or Open Resection Study Group, Buunen M, Veldkamp R, Hop WC, Kuhry E, Jeekel J, Haglind E, Påhlman L, Cuesta MA, Msika S, Morino M, et al: Survival after laparoscopic surgery verse open surgery for colon cancer: long-term outcome of a randomized controlled trial. Lancet Oncol 10: 44-52, 2009.

19. Cammà C, Giunta M, Fiorica F, Pagliaro L, Craxì A and Cottone M: Preoperative radiotherapy for resectable rectal cancer: a meta-analysis. JAMA 284: 1008-1015, 2000.

20. Kosmider S and Lipton L: Adjuvant therapies for colorectal cancer. World J Gastroenterol 13: 3799-3805, 2007.

21. Prosnitz RG, Patwardhan MB, Samsa GP, Mantyh CR, Fisher DA, McCrory DC, Cline KE, Gray RN and Morse MA: Quality measures for the use of adjuvant chemotherapy and radiation therapy in patients with colorectal cancer: a systematic review. Cancer 107: 2352-2360, 2006.

22. Machiels JP, Sempoux C, Scalliet P, et al: Phase I/II study of preoperative cetuximab, capecitabine, and external beam radiotherapy in patients with rectal cancer. Ann Oncol 18: 738-744, 2007.

23. Kapiteijn E, Marijnen CA, Nagtegaal ID, Putter $\mathrm{H}$ Steup WH, Wiggers T, Rutten HJ, Påhlman L, Glimelius B, van Krieken $\mathrm{JH}$, et al; Dutch Colorectal Cancer Group: Preoperative radiotherapy combined with total mesorectal excision for resectable rectal cancer. N Engl J Med 345: 638-646, 2001.

24. Gérard JP, Conroy T, Bonnetain F, Bouché O, Chapet O, Closon-Dejardin MT, Untereiner M, Leduc B, Francois E, Maurel J, et al: Preoperative radiotherapy with or without concurrent fluorouracil and leucovorin in T3-4 rectal cancers: Results of FFCD 9203. J Clin Oncol 24: 4620-4625, 2006.
25. Choy PY, Bissett IP, Docherty JG, Parry BR and Merrie AE: Stapled versus handsewn methods for ileocolic anastomoses. Cochrane Database Syst Rev 18: CD004320, 2007.

26. Gianotti L, Morelli L, Galbiati F, Rocchetti S, Coppola S, Beneduce A, Gilardini C, Zonenschain D, Nespoli A and Braga M: A randomized double-blind trial on perioperative administration of probiotics in colorectal cancer patients. World J Gastroenterol 16: 167-175, 2010.

27. Lustosa SA, Matos D, Atallah AN and Castro AA: Stapled versus handsewn methods for colorectal anastomosis surgery: a systematic review of randomized controlled trials. Sao Paulo Med J 120: 132-136, 2002.

28. Moran B and Heald R: Anastomotic leakage after colorectal anastomosis. Semin Surg Oncol 18: 244-248, 2008.

29. Walker KG, Bell SW, Rickard MJ, Mehanna D, Dent OF, Chapuis PH and Bokey EL: Anastomotic leakage is predictive of diminished survival after potentially curative resection for colorectal cancer. Ann Surg 240: 255-259, 2004.

30. Arguedas MR, Abrams GA, Krowka MJ and Fallon MB: Prospective evaluation of outcomes and predictors of mortality in patients with hepatopulmonary syndrome undergoing liver transplantation. Hepatology 37: 192-197, 2003.

31. Lertsithichai P and Rattanapichart P: Temporary ileostomy versus temporary colostomy: a meta-analysis of complications. Asian J Surg 27: 202-210, discussion 211-212, 2004.

32. Juneja V, Keegan P, Gootenberg JE, Rothmann MD, Shen YL, Lee KY, Weiss KD and Pazdur R: Continuing reassessment of the risks of erythropoiesis-stimulating agents in patients with cancer. Clin Cancer Res 14: 3242-3247, 2008.

33. Tsikitis VL, Malireddy K, Green EA, Christensen B, Whelan R, Hyder J, Marcello P, Larach S, Lauter D, Sargent DJ, et al: Postoperative surveillance recommendations for early stage colon cancer based on results from the clinical outcomes of surgical therapy trial. J Clin Oncol 27: 3671-3676, 2009.

34. Ickx BE, Bepperling F, Melot C , et al: wnormovolaemic haemodilution. Br J Anesth 91: 196-202, 2003. 